\title{
AN AUTOMATED TECHNIQUE FOR THE MEASUREMENT OF PEROXYACETYLNITRATE IN AMBIENT AIR AT PPB AND PPT LEVELS*
}

\author{
K. P. MÜLLER and J. RUDOLPH \\ Institut für Atmosphärische Chemie, Kernforschungsanlage Jülich, Postfach 1913, \\ D-S170 Jülich, FRG
}

(Received 27 October 1988; in final form 2 March 1989)

Peroxyacetylnitrate (PAN) is an important reaction product of photochemical air pollution. Due to its high toxicity and its importance as reservoir and iransport medium for nitrogen oxides, monitoring of PAN both in polluted and unpoiluted regions has become important. PAN levels in the background atmosphere may be as low as a few ppt. These low levels and the high reactivity and thermal instability of PAN require a very specialized technique if reliable monitoring of PAN is needed. A commercially available gaschromatograph (Sichromat 1) was equipped with pneumatically operated valves for automated column switching and air sample injection.

The system allows to optimize separation and detection of PAN at low temperatures of the column $\left(20^{\circ} \mathrm{C}\right)$ and the detector $\left(30^{\circ} \mathrm{C}\right)$, thus the thermal decomposition of PAN during separation and detection is avoided. Several "cut" and "backllush" steps eliminate the problem of column or detector contamination by other atmospheric pollutants. Measuring frequency is 6 per hour or better, the lower limit of detection is less than $5 \mathrm{ppt}$. To enable relaible, automated calibration in the field, a permeation device was developed which allows the generation of gas mixtures with PAN concentrations in the ppt and $\mathrm{ppb}$ range. A comparison of the permeation system with the injection of liquid PAN showed that both calibration methods gave the same results within the error of the measurements.

KEY WORDS: Peroxyacetylnitrate (PAN), ambient air, gaschromatography, automation, photochemical reactions

\section{INTRODUCTION}

Peroxyacetylnitrate (PAN) is formed as a product of the photochemical oxidation of organic trace gases in the atmosphere in the presence of nitrogen dioxide. Due to its toxicity and its importance for the photochemical reaction cycles in the atmosphere measurements of peroxyacetylnitrate in polluted and unpolluted regions are of considerable interest. Quite a number of measurement techniques and results of measurements have been published. ${ }^{1-15}$ Essentially all measurements of PAN in the atmosphere are made by gaschromatography with electron capture detection. In this paper we present an automated, quasi continuously operating gaschromatograph for PAN measurements at remote or semiremote stations. We

*Presented at the 18th International Symposium on Environmental and Analytical Chemistry, Barcelona, 5-8 September 1988. 
Figure-1 Schematic drawing of gaschromatograph and injection system. R: pressure regulators, a-d: pneumatic valves, 1: ten-way valve with actuator, 2-4: lour-way valves with actuator. Precolumn: $0.3 \mathrm{~m} \times 2 \mathrm{~mm}$, glass, packed with $5 \%$ PEG 400 on Chromosorb WHP (80/100 mesh). Separation column: same as precolumn but $1 \mathrm{~m}$ long.

also describe a permeation system for generation of PAN calibration gases. The system can be combined with an automatic preconcentration procedure if measurements in the ppt or sub-ppt range are needed.

The basic concept of the instrument is similar to a technique described in a previous publication. ${ }^{6}$ It has been modified to allow automated calibration, the long-term stability of the detector response has been improved by an additional cutting procedure which reduces the problem of detector contamination by other atmospheric trace gases and the lower limit of detection has been improved to 5 ppt in a $15 \mathrm{~cm}^{3}$ (STP) air sample.

\section{INSTRUMENT DESCRIPTION}

A schematic drawing of the inlet system and the chromatograph is shown in Figure 1. The sample air is drawn through the inlet line with a high flow rate in order to minimize possible wall effects. In order to load the sample loop, the pneumatic valves (a) and (d) are opened, the ten-way valve (1) is switched into the 
"load/backflush" position (gas flow pattern is shown by dashed lines) with the four-way valve (2) in "open" position (solid lines). The flow controller maintains a constant flow of about $50 \mathrm{~cm}^{3} \mathrm{~min}^{-1}$ through the inlet system. Then valves (a) and (d) are closed and valve (c) is opened. Thus the sample air in the "compression tube", a teflon tube $50 \mathrm{~cm}$ length and $6 \mathrm{~mm}$ i.d., is pushed into the sample loop until the sample pressure is identical to the carrier gas pressure. This eliminates the pressure peak during injection and results in a well defined sample input profile.

Next, four-way valve (2) is closed and the ten-way valve rotated into "inject" position (solid lines). The sample is injected by opening four-way valve (2). After preseparation on the precolumn, the light fraction of the sample, including oxygen, is vented via four-way valve (3). The needle valve is used to keep the gas pressure at the end of the precolumn constant, independent of the position of four-way valve (3). PAN is transferred to the main separation column by rotating valve (3). Then the precolumn is flushed back. This prevents water and trace constituents of low volatility entering the main column.

Due to the venting of the low boiling substances and the backflush of the heavier fraction, essentially only PAN enters the electron capture detector. In Figure 2 two chromatograms of PAN in outside air are shown. Figure 2a shows a chromatogram without venting oxygen, $2 b$ with. Evidently this not only reduces the oxygen peak (retention time about $1 \mathrm{~min}$ ) but also eliminates the baseline drift between 4 and 7 minutes retention time. As can be seen, the size of the PAN-peak is not affected. Due to the "cut" and the "backflush" steps both the detector and the columns can be operated at rather low temperatures without baseline drift

ssure regulators, a-d: actuator. Precolumn: 10 mesh). Separation which might be caused by the accumulation of other trace substances in the detector or column. The column oven is operated at $293 \mathrm{~K}$. For operation near or even below ambient temperature the column oven is equipped with a heat exchanger which is cooled by a cryostat. The detector is kept at $300 \mathrm{~K}$. In this :ation gases. The cedure if measurtemperature range, the ECD response for PAN varies only slightly with temperature, about $2 \%$ if the temperature is raised by $5 \mathrm{~K}$. However above $320 \mathrm{~K}$ the detector sensitivity decreases rapidly with increasing temperature, about $30 \%$ if the temperature is raised from $320 \mathrm{~K}$ to $335 \mathrm{~K}$. For optimum detector sensitivity and stability, the ECD-temperature should be maintained below $310 \mathrm{~K}$. To further improve the signal to noise ratio, the ECD is contained in a small housing which is flushed with nitrogen. This reduces the baseline noise caused by oxygen diffusion into the detector cell. The lower limit of detection for PAN is about $5 \times 10^{-13} \mathrm{~g}$. This corresponds to a volume mixing ratio of $5 \mathrm{ppt}$ in a $15 \mathrm{~cm}^{3}$ (STP) air sample. The injection of substantially larger air samples would result in a significant broadening of the PAN peak. The reproducibility of the measurements is about $5 \%$, long-term stability (several weeks) of the instrument is better than $15 \%$. The maximum sampling rate is about one measurement every 8 minutes.

In Figure 3 we show the diurnal cycles of the PAN mixing ratio in a semirural aph is shown in high flow rate in sample loop, the switched into the area near Jülich in West Germany. It can be seen that the measurement frequency and the reproducibility of the method allows the resolution of detailed structures of the diurnal variation of PAN. 


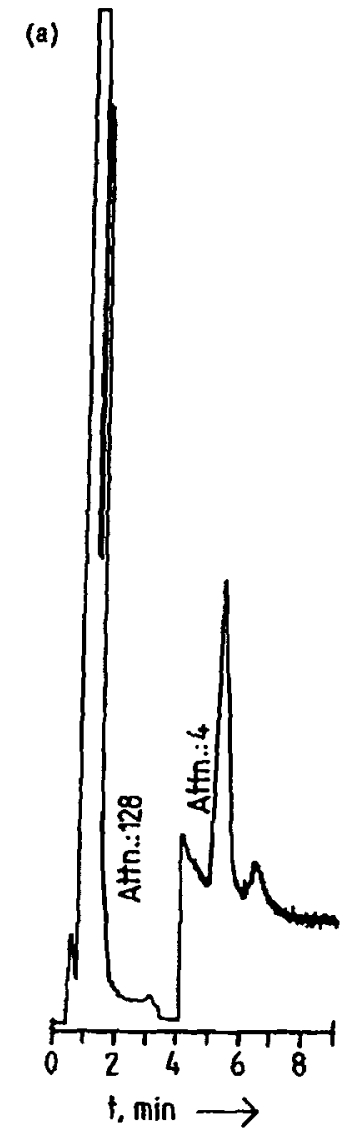

Figure 3 Diurnal cy

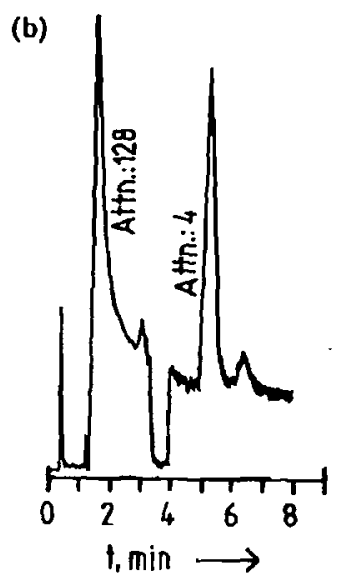

Central European Su

\section{CAI.IBRATION}

A few years ago organic solvents । procedure very si of PAN in the si hydroxide solutio ECD response, $d r$ by injection of a solution. This is $c$ way valve (4) in test or calibrate $t$ mixing ratios in Figure 4. Nitroge piece of teflon tut

Figure 2 Two chromatograms of air samples $\left(5 \mathrm{~cm}^{3}\right)$ measured within $20 \mathrm{~min}$. The PAN peak (retention time $5 \mathrm{~min}$ ) corresponds to $0.3 \mathrm{ppb}$ volume mixing ratio. a: without venting oxygen and the to obtain a low : low boiling fraction of the sample. b: with venting. 

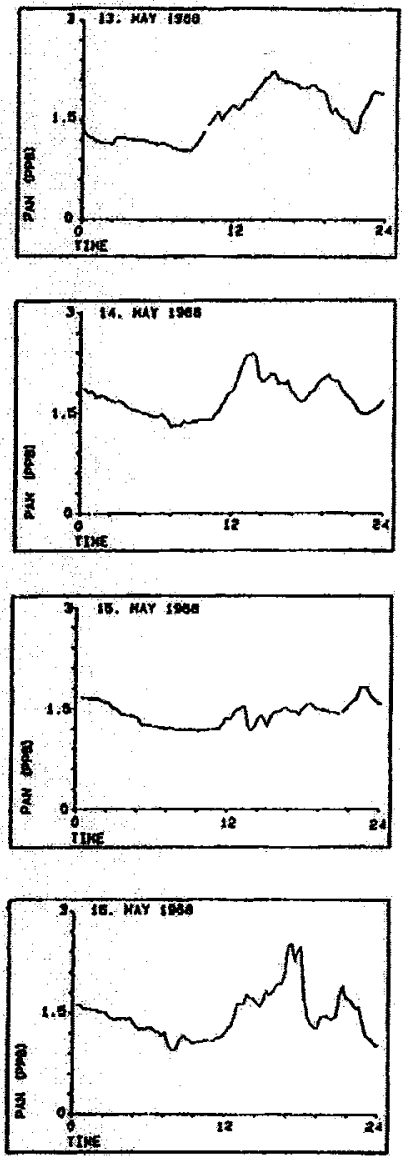

Figure 3 Diurnal cycles of PAN, measured during a campaign in May 1988. The time shown is Central European Summertime.

\section{CALIBRATION}

A few years ago procedures for the preparation of pure peroxyacetylnitrate in organic solvents were developed. We prepared solutions of PAN in $n$-heptane by a procedure very similar to the one described by Nielson et al. ${ }^{16}$ The concentration of PAN in the solution was measured by hydrolysis with dilute aqueous sodium hydroxide solutions and subsequent photometric measurement of the nitrite ions. ECD response, detector linearity, retention time of PAN etc. can easily be checked by injection of a few $\mu \mathrm{l}$ of $n$-heptane containing some $\mu$ l of PAN per $\mathrm{dm}^{3}$ of solution. This is done by means of a commercial liquid injector (see Figure 1, fourway valve (4) in "open" position (dashed lines)). However this does not allow to test or calibrate the gas injection system. For the generation of gaseous PAN with mixing ratios in the ppb and ppt range we use the permeation device shown in Figure 4. Nitrogen with a flow rate of about $50 \mathrm{~cm}^{3} \mathrm{~min}^{-1}$ is passed through a piece of teflon tubing immersed in a dilute solution of PAN in $n$-heptane. In order to obtain a low and constant permeation rate and to increase the stability of the

10 min. The PAN peak yenting oxygen and the 
K. P. MULLLER AND J. RUDOLPH
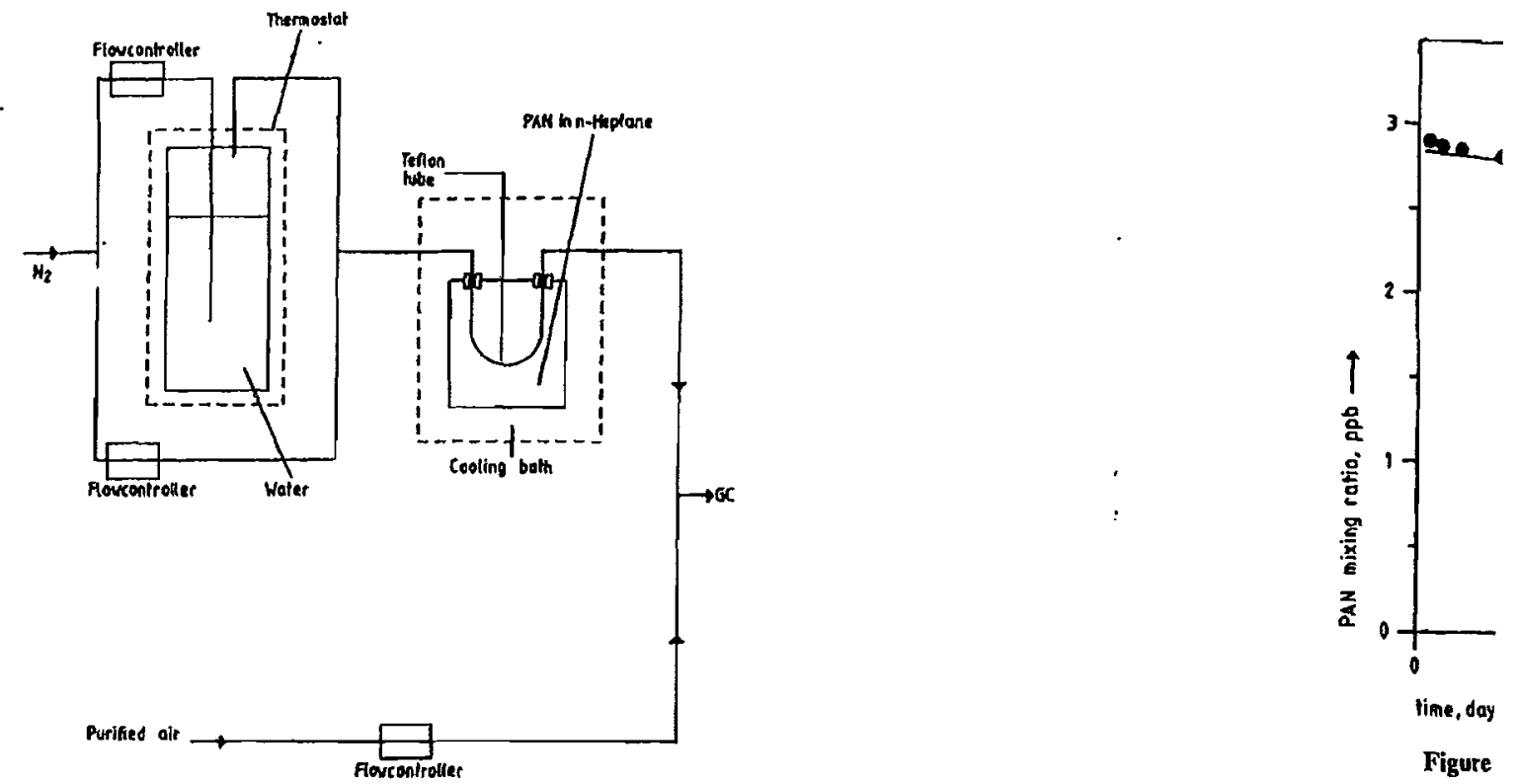

Figure

Figure 4 Schematic drawing of permeation system for the generation of calibration gases with PAN mixing ratios in tho $\mathrm{ppb}$ and $\mathrm{ppt}$ range.

solution, the permeation device is kept at $275 \mathrm{~K}$. In order to cnable tests with moist gases, the gas, or part of it, can be passed through a bubbler filled with deionized water at a defined temperature.

The calibration gas can be further diluted with purified air. This allows calibrations and tests covering about 3 orders of magnitude of PAN mixing ratios. The mixing ratio of PAN in the calibration gas is measured by passing it through. a dilute aqueous sodium hydroxide solution and photometric measurement of $\mathrm{NO}_{2}^{-}$, one of the products of PAN hydrolysis in alkaline solutions.

Without further dilution, the permeation device generates nitrogen with PAN mixing ratios of a few $\mathrm{ppb}$. In Figure 5 the change in the PAN mixing ratio with time is shown. It can be seen that the change within one month is less than $10 \%$. This allows a reliable calibration of the gaschromatograph over longer periods of time. Automated calibration can be made by injection of the calibration gas into the chromatograph instead of outside air (pneumatic valve (b) open instead of (a), Figure 1). This can be made regularly even during unattended operation. A comparison between calibration by injection of PAN in solution and the permeation device showed that both methods agree within an error of $10 \%$. This is within the total errors of the measurements.

\section{PRECONCENTRATION}

With a lower limit of detections of about $5 \mathrm{ppt}$, the described measuring procedure is sufficiently se: urban areas. $\mathrm{HC}$ mixing ratios of the directly inje step is necessar PAN can be at (195 K). ${ }^{6}$ For cr is not acceptabl immersion cool vessel we use th trap. For precc "vent" position. chamber is equi sample loop (gl components of sample loop. $F$ "pressurize" po: replaced by pre hydrostatic pre The temperatur is heated to roc desorption und due to thermal system as show 


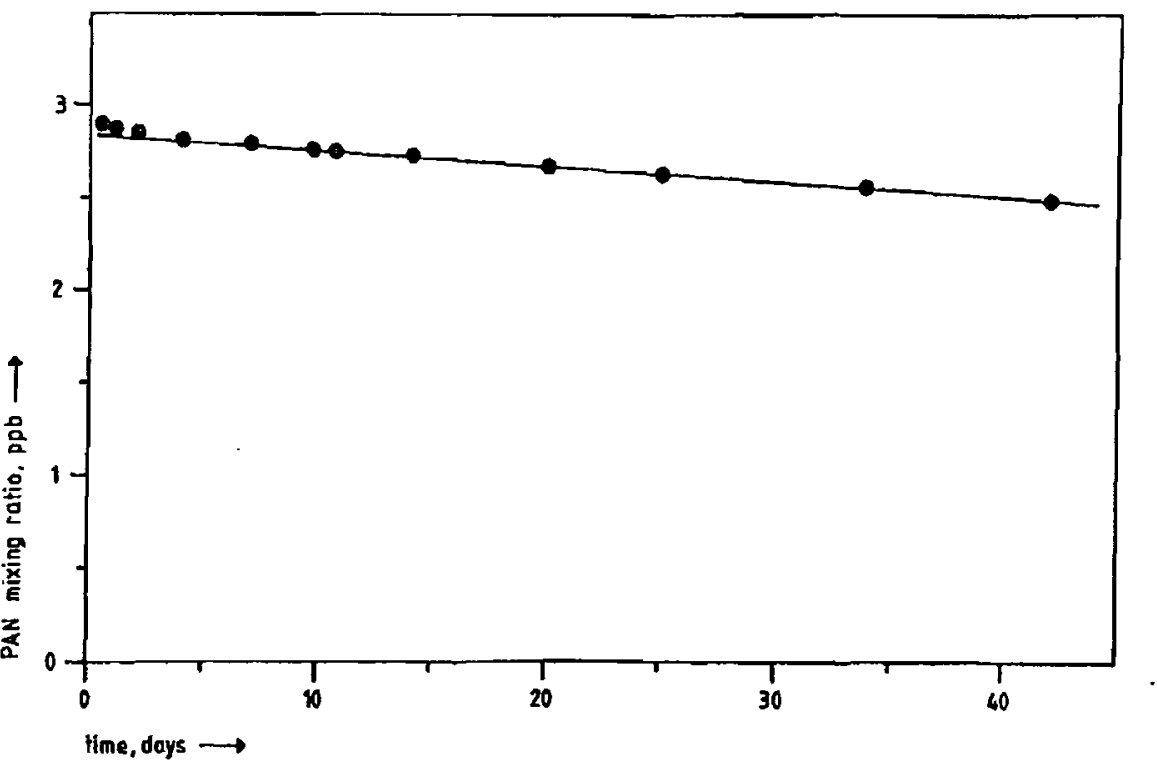

Figure 5 Variation with time of the PAN mixing ratio in the calibration gas.

ibration gases with PAN

to enable tests with bubbler filled with

id air. This allows PAN mixing ratios. $y$ passing it through ric measurement of ions.

nitrogen with PAN $\mathrm{N}$ mixing ratio with th is less than $10 \%$. 'er longer periods of calibration gas into open instead of (a); ended operation. A ion and the permea$: 10 \%$. This is within

measuring procedure is sufficiently sensitive for measurements in most continental regions, even outside urban areas. However in extremely remote areas and in the maritime atmosphere mixing ratios of PAN below $10 \mathrm{ppt}$ have been observed.,11 Since the volume of the directly injected sample is limited to about $15 \mathrm{~cm}^{3}$ (STP) a preconcentration step is necessary if larger sample volumes are needed. It has been reported that PAN can be absorbed on the walls of a glass tube which is cooled with dry ice $(195 \mathrm{~K}){ }^{6}{ }^{6}$ For continuous unattended measurements the use of dry ice for cooling is not acceptable. We use an isopropanol bath which is cooled with a two-stage immersion cooler instead. In order to avoid the mechanical moving of a dewar vessel we use the system shown in Figure 6 for cooling and heating the enrichment trap. For preconcentration of PAN the three-way solenoid valve is switched to "vent" position. Then the level of the cooling bath $(180 \mathrm{~K})$ in the preconcentration chamber is equal to the level in the dewar vessel. Outside air is drawn through the sample loop (glass tube of $30 \mathrm{~cm}$ length, $4 \mathrm{~mm}$ i.d.). PAN and other atmospheric components of low volatility are quantitatively adsorbed at the surface of the sample loop. For desorption the three-way solenoid valve is switched to the "pressurize" position and the cooling liquid in the preconcentration chamber is replaced by pressurized air. The air pressure is adjusted precisely to balance the hydrostatic pressure of the cooling liquid outside the preconcentration chamber. The temperature of the sample loop is increased to $280 \mathrm{~K}$ by circulating air which is heated to room temperature through the preconcentration chamber. This allows desorption under very moderate conditions which is essential to avoid PAN losses due to thermal decomposition. The sample loop is connected to the sample inlet system as shown in Figure 1. During desorption the four-way valve (2) is closed 


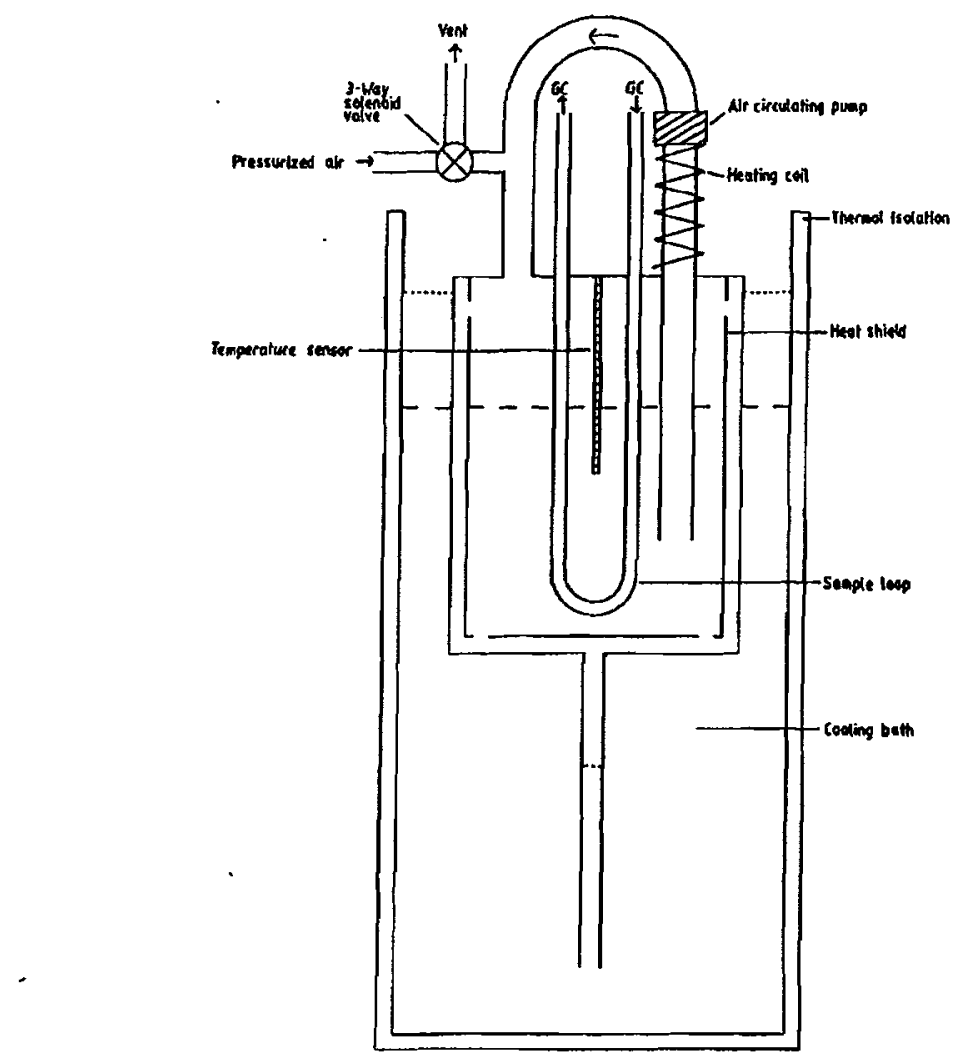

Figure 6 Schematic drawing of the preconcentration device. Details see text.

(dashed lines). After desorption the four-way valve is rotated and the desorbed sample injected as a delined, small volume.

The chromatogram in Figure 7 shows the separation of PAN from a sample preconcentrated from $300 \mathrm{~cm}^{3}$ (STP) of ambient air. The PAN mixing ratio was about $0.5 \mathrm{ppb}$. As can be seen from the size of the PAN peak (retention time $5 \mathrm{~min}$, PAN peak attenuated by a factor of 64 , baseline attenuated by only a factor of 4) and the noise of the baseline, measurements of PAN mixing ratios below 1 ppt are possible with the help of such a preconcentration step. The time needed for the preconcentration procedure is between 5 and 15 minutes (flowrates of about $50 \mathrm{~cm}^{3} \mathrm{~min}^{-1}$ (STP) and total sample volumes from $250-750 \mathrm{~cm}^{3}$ ). Including separation and desorption of the preconcentrated sample the total time for one measurement is between 20 and $30 \mathrm{~min}$. Within the uncertainty of the measurements $(\approx 10 \%)$, adsorption and recovery of PAN is quantitative.

\section{CONCLUSIONS}

The described method enables routine, unattended measurements of PAN with a

Figure 7 Chromato (5min retention time PAN peak is attenua tentatively identilied

time resolution ، and "backflush" limit of detectio allows long tern detection for $P A$ sample. Measurt ppt range) req procedure is suil samples every he

Calibration o system for PAN instrument can I 
?AN from a sample N mixing ratio was seak (retention time ited by only a factor ixing ratios below 1 The time needed for (llowrates of about $\left.-750 \mathrm{~cm}^{3}\right)$. Including z total time for one nty of the measure'e.
\end{abstract}

ents of PAN with a

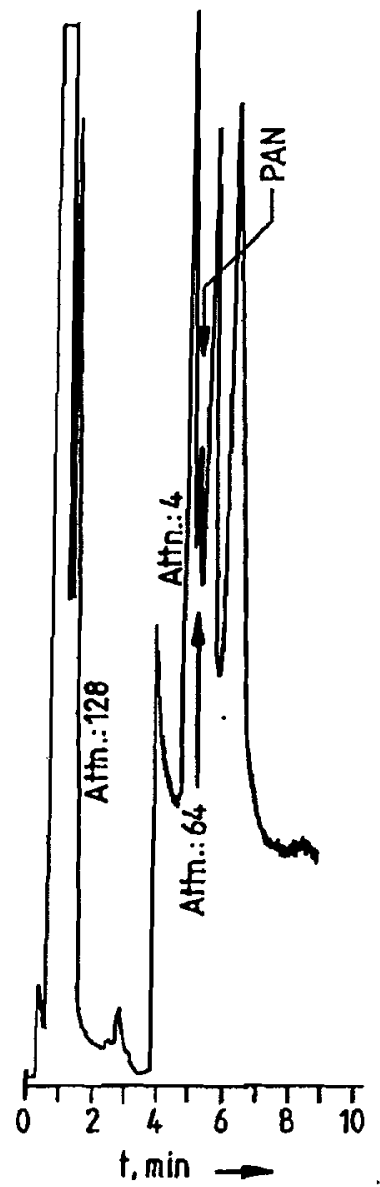

Figure 7 Chromatographic separation of a preconcentrated sample $\left(300 \mathrm{~cm}^{3}, S T P\right)$. The PAN peak ( 5 min retention time) corresponds to a mixing ratio of $0.5 \mathrm{ppb}$. It should be noted that the top of the PAN peak is attenuated by a factor of 64 whereas the baseline below the PAN peak and the next peak, tentatively identified as peroxypropionyl nitrate is only attenuated by a factor of 4 .

time resolution of $10 \mathrm{~min}$ at mixing ratios in the sub ppb range. The use of "cut" and "backflush" steps not only reduces the analysis time and gives a better lower limit of detection, but also improves the stability of the detector response and allows long term unattended operation at remote stations. The lower limit of detection for PAN is $5 \times 10^{-13} \mathrm{~g}$ which is equivalent to about $5 \mathrm{ppt}$ in a $15 \mathrm{~cm}^{3}$ sample. Measurements of PAN at lower mixing ratios (lowest ppt range and sub ppt range) require a preconcentration step. The presented preconcentration procedure is suitable for automated operation at a measuring frequency of up to 2 samples every hour.

Calibration of the instrument can be made with the described permeation system for PAN on a routine basis, if necessary a regular calibration check of the instrument can be made automatically. 
We have successfully used the presented method during a 5 week period in May 1988 and have just now started to measure the latitudinal distribution of PAN with an instrument on board of the German research vessel F.S. Polarstern.

\section{Acknowledgement}

TEMPORA] SPECIFIC

This work was supported financially by the Bundesminister für Forschung und Technologie of the Federal Republic of Germany.

JOACl

Atmospheric Resear. Protection

\section{References}

1. E. R. Stephens, Adv. Envir. Sci. 1, 119 (1969).

2. S. A. Penkett, F. J. Sandalls and J. G. Lovelock, Atmos. Environ. 9, 139 (1975).

3. H. B. Singh and L. J. Salas, Atmos. Environ. 17, 1507 (1983).

4. H. B. Singh and L. J. Salas, Nature 302, 326 (1983).

5. H. Nieboer and J. van Ham. Atmos. Environ. 10, 115 (1976),

6. B. Vierkorn-Rudolph, J. Rudolph and S. Diederich, Intern J. Environ. Anal. Chem. 20, 131 (1985).

7. C. W. Spicer, M. W. Holdren and G. W. Keigley, Atmos. Environ. 17, 1055 (1983).

8. T. Nielsen, U. Samuelsson, P. Grennfelt and E. L. Thomsen, Nature 293, 553 (1981).

9. E. R. Stephens, P. L. Hanst, R. C. Doerr and W. E. Scott, Ind. Engng. Chem. 48, 1498 (1956).

10. W. A. Lonnemann, J. J. Bufalini and R. L. Seila, Environ. Sci. Technol. 10, 374 (1976).

11. J. Rudolph, B. Vierkom-Rudolph and F. X. Meixner, J. Geophys. Res. 92, 6653 (1987).

12. J. W. Bottenheim, A. G. Gallant and K. A. Brice, Geophys. Res. Lett, 13, 113 (1986).

13. K. A. Brice, S. A. Penkett, D. H. F. Atkins, F. J. Sandalls, D. J. Bamber, A. F. Tuck and G. Vaughn, Atmos. Environ, 18, 2691 (1984).

14. H. B. Singh, L. J. Salas and W. Viezee, Nature, 321, 588 (1986).

15. D. W. Fahey, G. Hübler, D. D. Parrish, E. J. Williams, R. B. Norton, B. A. Ridley, H. B. Singh, S. C. Lin and F. C. Fehsenfeld, J. Geophys. Res. 91, 9781 (1986).

16. T. Nielsen, A. M. Hansen and E. L. Thomsen, Atmos. Environ. 16, 2447 (1982). collected samples are th
NSI Environm

\section{$($.}

Methodology was develo! they vary in concentratic organic compounds (VOr exposures to insignificant syringe sampler that can separation using mass $\mathrm{s}$ representative results an concentrations in situatic automobile indoor air du The method is shown to and sample integrity test tests show that VOC cor scales, ranging from $2 \mathrm{mil}$

KEY WORDS: Indoor

\section{INTRODUCTION}

The identification : (VOCs) in the bre: compounds have be techniques are in $u s$ which rely on time collection of whole-i addition, semi-real-1 instruments have $b$ only as screening tc

*To whom correspond 